\title{
First Physics Results from ALICE
}

\author{
Iouri BELIKOV* for the ALICE Collaboration \\ IPHC, Université de Strasbourg, CNRS-IN2P3 \\ E-mail: iouri.belikov@in2p3.fr
}

\begin{abstract}
We will present the results from the first series of measurements performed by the ALICE experiment at CERN LHC. The results include the charged-particle pseudorapidity densities, multiplicity and transverse momentum distributions obtained by analysing the pp data collected in 2009 and 2010 at three different center-of-mass energies of 0.9, 2.36 and $7 \mathrm{TeV}$. Compared to previous proton-antiproton data and to model predictions, these results provide insights about the mechanisms of particle production in pp collisions at the highest available so far energies. They will serve as a baseline for the future measurements at even higher LHC energies in pp, and in heavy-ion collisions.
\end{abstract}

35th International Conference of High Energy Physics

July 22-28, 2010

Paris, France

${ }^{*}$ Speaker. 


\section{Introduction}

A Large Ion Collider Experiment (ALICE) [1] at CERN is a general-purpose heavy-ion experiment designed to study the physics of strongly interacting matter in nucleus-nucleus collisions at the LHC. These studies cannot be effectively performed without having a reliable "hadronic reference". All the measurements that will be done by ALICE in heavy-ion collisions will have to be compared with the corresponding, properly scaled, proton-proton results. Also, it is important to check if the models that successfully described the particle production in "elementary" collisions at lower energies still do so when extrapolated to the LHC energy domain. Finally, one might expect something completely new (like some kind of collective effects at the partonic level) to happen in pp collisions at these new LHC energies. For this, we would need to study all the observables as the function of multiplicity. Which, in turn, requires good multiplicity measurements.

The description of the ALICE detectors can be found in [1]. As for the first pp runs in 2009 and 2010, the most important detectors are the Inner Tracking System (ITS), the Time Projection Chamber (TPC), the Time-Of-Flight detector (TOF) (all covering the pseudorapidity range $|\eta|<$ 0.9 ), and the muon spectrometer (MUON) (with the pseudorapidity coverage $-4<\eta<-2.5$ ).

The triggering for the first collisions is essentially minimum bias. At least one charged particle is required in about 8 units of pseudorapidity covered by the two innermost pixel layers of the ITS (covering the ranges $|\eta|<2.0$ and $|\eta|<1.4$ ) and the two scintillating rings of the V0A and the V0C detectors (with the coverage of $2.8<\eta<5.1$ and $-3.7<\eta<-1.7$ ). A special single-muon trigger is also implemented for triggering the muon spectrometer, and it is read out in coincidence with the general minimum-bias trigger. The coincidence with the beam pickup counters, in several logical combinations, allows for accepting special control triggers used for the background estimations.

We have taken data at three energies: $0.9 \mathrm{TeV}, 2.36 \mathrm{TeV}$ and $7 \mathrm{TeV}$. At 0.9 and $2.36 \mathrm{TeV}$ we consider two event classes: inelastic (INEL) and non-single-diffractive (NSD). The corresponding corrections for the triggering efficiency are done by tuning the MC event class fractions to match the UA5 [2], E710 [3] and CDF [4] data, and using PYTHIA 6.4.14 and 6.4.21 [5, 6], tune D6T [7], and PHOJET 1.12 [8] Monte Carlo models to assess the effects of different kinematics. At $7 \mathrm{TeV}$, we use the hadron-level event class definition, requiring at least one charged particle in a pseudorapidity window $|\eta|<1$.

\section{The first physics results}

Charged-particle pseudorapidity density and multiplicity distributions. The first multiplicity measurements in ALICE were done with the two inner most pixel layers of the ITS. ALICE has published the charged-particle pseudorapidity $\left(\mathrm{d} N_{\mathrm{ch}} / \mathrm{d} \eta\right)$ densities and multiplicity distributions obtained for the INEL and NSD events in pp collisions at $0.9 \mathrm{TeV}, 2.36 \mathrm{TeV}$ and $7 \mathrm{TeV}[9,10,11]$. The results were found to be consistent with UA5 p p data at $0.9 \mathrm{TeV}[12,13]$, and also with CMS pp data at 0.9 and $2.36 \mathrm{TeV}$ [14] (for the NSD events).

The obtained multiplicity distributions fit well to a single negative binomial distribution, and the distribution for the INEL events tends to have more events with low multiplicity than the NSD events do. The dependence of $\mathrm{d} N_{\mathrm{ch}} / \mathrm{d} \eta$ on the collision energy $\sqrt{s}$ is well described by the power- 

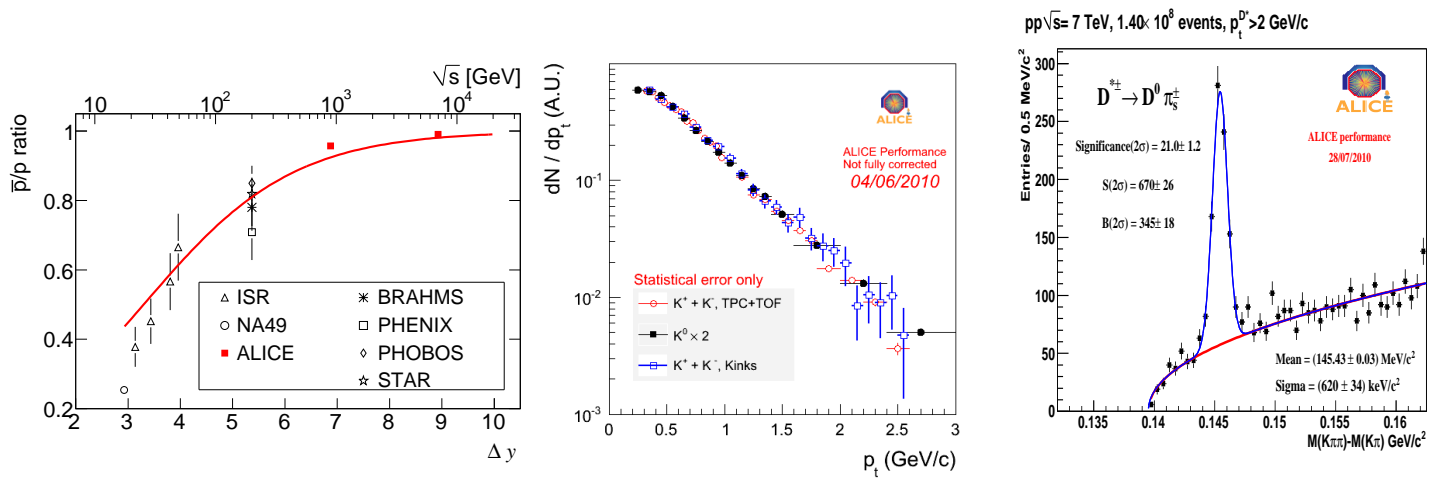

Figure 1: Left: Mid-rapidity $\overline{\mathrm{p}} / \mathrm{p}$ ratio as a function of the rapidity interval $\Delta y$ and center-of-mass energy $\sqrt{s}$ [15]. Middle: Not fully corrected transverse-momentum spectrum of charged kaons identified with TPC and TOF and reconstructed via the kink decay topology, overlapped on the spectrum of $\mathrm{K}_{\mathrm{S}}^{0}$ reconstructs via the V0 decay topology. Right: Invariant mass spectrum of charged $\mathrm{D}^{*}$ mesons identified in $\mathrm{D}^{0} \pi$ decay channel.

law function $\left(\sim s^{0.1}\right)$ [11]. This increase with $\sqrt{s}$ is systematically bigger than predicted by several PYTHIA tunes and PHOJET.

Mid-rapidity anti-proton to proton ratio. This measurement provides additional an important piece information for the theoretical discussion over the question: what carries the baryon number? The models postulating that the baryon number is carried by valence di-quarks, predict the $\overline{\mathrm{p}} / \mathrm{p}$ ratio should be 1 at the LHC energies. Other models involve special configurations of gluonic field, the so called "string junctions", that may also be related to the baryon number (see, for example, the references in [15]). In these models, the $\bar{p} / p$ ratio does not reach 1 even at the LHC energies.

ALICE has performed this measurement and has added two important points to the the $\bar{p} / \mathrm{p}$ excitation function (see Fig. 1, left). At $7 \mathrm{TeV}$, the measured value of $\bar{p} / \mathrm{p}$ is $0.990 \pm 0.006 \pm 0.014$ (the first error is statistical, the second is systematic). The ratio has also been studied as the function of the transverse momentum. It has been found that the models with string junctions systematically underestimate the data, especially at $0.9 \mathrm{TeV}$ [15].

Altogether, these results put a strong constraint on the association between the string junctions and the baryon number.

Bose-Einstein correlations with charged pions. The Bose-Einstein correlations of identical pions are a well known tool to evaluate the size and the space-time evolution of the emitting system. ALICE has measured the corresponding correlation function in pp collisions at $0.9 \mathrm{TeV}$ as the function of $q_{\text {inv }}=\left|\mathbf{p}_{1}-\mathbf{p}_{2}\right|$ ( $\mathbf{p}_{1}$ and $\mathbf{p}_{2}$ being the momenta of the pions in a pair) [16].

The radius of the emitting system grows with the event multiplicity, consistently with the measurements by other experiments. At the same time, it stays approximately constant as the function of the absolute value of the difference between the transverse momenta of the pions in a pair, $k_{\mathrm{T}}=\left|\mathbf{p}_{1, \mathrm{~T}}-\mathbf{p}_{2, \mathrm{~T}}\right| / 2$, which is different from the previous observations. We also report a significant systematic uncertainty inevitably affecting all these measurements. This systematic uncertainty comes from the assumption about the shape of the "base line", which is, likely, not to be flat in pp, as it is often assumed. 
Particle production, transverse-momentum spectra, jets and high- $p_{\mathrm{T}}$ particle correlations. The charged-particle transverse-momentum spectrum measured by ALICE in the pseudorapidity window $|\eta|<0.8$ [17] fits well to the modified Hagedorn function. At the transverse momenta $p_{\mathrm{T}}>3 \mathrm{GeV} / c$, the shape of the spectrum is somewhat better described by the power-law function. The comparison to PHOJET and different PYTHIA tunes shows that none of these Monte Carlo models gives a reasonable description of the data.

We also compare this spectrum with the ones measured by other experiments (UA1, ATLAS and CMS, see the references in [17]). ALICE's spectrum is harder. This has been understood as the consequence of the narrower pseudorapidity window that is used by ALICE.

A rather strong test of the available models can be performed by looking at the correlation between the mean $p_{\mathrm{T}}$ and the multiplicity. It turns out that none of the tried Monte Carlo models is able to simultaneously reproduce the measured correlation [17] and the multiplicity distributions reported in [10].

At the moment of presenting these results (July 2010), the transverse-momentum spectra of identified particles $\left(\pi^{ \pm}, \mathrm{K}^{ \pm}, \mathrm{p}^{ \pm}, \mathrm{K}_{\mathrm{S}}^{0}, \Lambda^{0}, \bar{\Lambda}^{0}\right.$ and $\left.\Xi^{ \pm}\right)$are not yet fully normalized. However, all the ALICE detectors contributing to these measurements (ITS, TPC and TOF) have already reached a high level of calibration, the evaluation of the involved corrections is almost done, and so the results are very close to the final ones. This is illustrated by the Fig. 1 (middle) that shows the not fully corrected transverse-momentum spectrum of kaons reconstructed by three completely different methods: particle identification using the TPC and TOF, a kink decay topology reconstruction (for charged kaons) and the V0 decay topology reconstruction (for $\mathrm{K}_{\mathrm{S}}^{0}$ ).

Quite a few items in ALICE's physics programme require the detection of resonances, the $\mathrm{K}^{* 0}$ and $\phi$ in particular. These resonances are clearly seen by our detectors and their parameters (mass and width) are consistent with the corresponding PDG values.

The $\pi^{0}$ and $\eta$ mesons can be detected in ALICE by means of double $\gamma$-conversion reconstruction in TPC and ITS, and also using the calorimeters, PHOton Spectrometer (PHOS) and ElectroMagnetic CALorimeter (EMCAL). The reconstruction of the $\gamma$ conversions is also used for a precise mapping of the material distribution in the detectors.

ALICE is also capable of reconstructing the jets and measuring the jet spectra. The collaboration is working on the correlations between the leading (high- $p_{\mathrm{T}}$ ) particles and other particles in the event. Once the leading particles are defined and isolated, one can study the properties of the underlying event, which is sensitive to the soft-component and multi-parton interactions. Our data show significantly more activity in the underlying event than what is predicted by the usual Monte Carlo models, especially when the momentum of the leading particle is below $10 \mathrm{GeV} / c$.

Heavy-flavour particle measurements. With the statistics that we have at the moment of the conference $\left(\sim 1.5 \times 10^{8}\right.$ minimum bias pp events at $\left.7 \mathrm{TeV}\right)$, we can already see clear signals of $\mathrm{J} / \psi$ and $\mathrm{D}$ mesons. The subsequent extraction of the $p_{\mathrm{T}}$ spectra of these particles has started.

In the mid-rapidity region of ALICE, we can measure the $\mathrm{J} / \psi$ spectrum reconstructed in the $e^{+} e^{-}$decay channel. In the forward rapidity region, the same spectrum can also be obtained in the $\mu^{+} \mu^{-}$decay channel, using the MUON spectrometer. In both cases, this spectrum can be measured down to $p_{\mathrm{T}} \sim 0$.

The $\mathrm{D}$ meson family can be reconstructed in several decay channels. The $\mathrm{D}^{0}$ meson is easily detected in the $\mathrm{K} \pi$ decay channel. In addition to $\mathrm{D}^{0}$ 's, we also reconstruct the charged $\mathrm{D}$ mesons in 
the $\mathrm{K} \pi \pi$ channel, and even the charged $\mathrm{D}^{*}$ mesons in the $\mathrm{D}^{0} \pi$ channel, as shown in Fig. 1 (right). All the signals can be extracted in momentum bins and up to $p_{\mathrm{T}} \sim 15 \mathrm{GeV} / c$ at least.

We are also investigating the possibilities to study the charm and beauty production using the the semi-leptonic decay channels. Currently, in the mid-rapidity part of ALICE, we identify the electrons by the energy loss in the TPC. Soon, the Transition Radiation Detector and the PHOS and EMCAL will start contributing to the identification, which will then extend the accessible $p_{\mathrm{T}}$ range. Special measures are taken to suppress the background from the $\gamma$ conversions: selection the tracks having bigger impact parameters and having a cluster attached at the inner-most layer of the ITS, and subtracting from the final spectrum the electron spectrum associated with the reconstructed $\gamma$ 's.

In the MUON spectrometer, the single muon spectrum is corrected for the contribution of muons coming from other than heavy-flavour particle decays, using detailed Monte Carlo simulations with PYTHIA. The resulting spectrum is then separated into the charm and beauty contributions by fitting to the spectral shapes provided by PQCD calculations.

\section{Conclusions}

The ALICE experiment has successfully started taking and analyzing data recorded at $0.9,2.36$ and $7 \mathrm{TeV}$ in pp collisions at LHC. The obtained first results are valuable for better understanding the mechanisms of particle production at the LHC energies. These results are also important as the reference for ALICE's main stream studies that will start in nucleus-nucleus collisions at the end of this year.

\section{References}

[1] F. Carminati et al. [ALICE Collaboration], J. Phys. G 30, 1517 (2004).

[2] R. E. Ansorge et al. [UA5 Collaboration], Z. Phys. C 33 (1986) 175.

[3] N. A. Amos et al. [E710 Collaboration], Phys. Lett. B 301 (1993) 313.

[4] A. A. Affolder et al. [CDF Collaboration], Phys. Rev. Lett. 87 (2001) 141802.

[5] T. Sjostrand, Comput. Phys. Commun. 82 (1994) 74.

[6] T. Sjostrand, S. Mrenna and P. Z. Skands, JHEP 0605 (2006) 026.

[7] M. G. Albrow et al. [TeV4LHC QCD Working Group], arXiv:hep-ph/0610012.

[8] R. Engel, J. Ranft and S. Roesler, Phys. Rev. D 52 (1995) 1459.

[9] K. Aamodt et al. [ALICE Collaboration], Eur. Phys. J. C 65 (2010) 111.

[10] K. Aamodt et al. [ALICE Collaboration], Eur. Phys. J. C 68 (2010) 89.

[11] K. Aamodt et al. [ALICE Collaboration], Eur. Phys. J. C 68 (2010) 345.

[12] G. J. Alner et al. [UA5 Collaboration], Z. Phys. C 33 (1986) 1.

[13] R. E. Ansorge et al. [UA5 Collaboration], Z. Phys. C 43 (1989) 357.

[14] V. Khachatryan et al. [CMS Collaboration], JHEP 1002 (2010) 041.

[15] A. K. Aamodt et al. [ALICE Collaboration], Phys. Rev. Lett. 105 (2010) 072002.

[16] A. K. Aamodt et al. [ALICE Collaboration], Phys. Rev. D 82, (2010) 052001.

[17] K. Aamodt et al. [ALICE Collaboration], Phys. Lett. B 693 (2010) 53. 